\title{
NONCOMPLETE AFFINE STRUCTURES ON LIE ALGEBRAS OF MAXIMAL CLASS
}

\section{E. REMM and MICHEL GOZE}

Received 29 January 2001

\begin{abstract}
Every affine structure on Lie algebra $\mathfrak{g}$ defines a representation of $\mathfrak{g}$ in $\operatorname{aff}\left(\mathbb{R}^{n}\right)$. If $\mathfrak{g}$ is a nilpotent Lie algebra provided with a complete affine structure then the corresponding representation is nilpotent. We describe noncomplete affine structures on the filiform Lie algebra $L_{n}$. As a consequence we give a nonnilpotent faithful linear representation of the 3-dimensional Heisenberg algebra.
\end{abstract}

2000 Mathematics Subject Classification: 53Cxx, 17Bxx.

\section{Affine structure on a nilpotent Lie algebra}

\subsection{Affine structure on nilpotent Lie algebras}

DefinITION 1.1. Let $\mathfrak{g}$ be an $n$-dimensional Lie algebra over $\mathbb{R}$. An affine structure is given by a bilinear mapping

$$
\nabla: \mathfrak{g} \times \mathfrak{g} \longrightarrow \mathfrak{g}
$$

satisfying

$$
\begin{gathered}
\nabla(X, Y)-\nabla(Y, X)=[X, Y], \\
\nabla(X, \nabla(Y, Z))-\nabla(Y, \nabla(X, Z))=\nabla([X, Y], Z),
\end{gathered}
$$

for all $X, Y, Z \in \mathfrak{g}$.

If $\mathfrak{g}$ is provided with an affine structure, then the corresponding connected Lie group $G$ is an affine manifold such that every left translation is an affine isomorphism of $G$. In this case, the operator $\nabla$ is nothing but the connection operator of the affine connection on $G$.

Let $\mathfrak{g}$ be a Lie algebra with an affine structure $\nabla$. Then the mapping

$$
f: \mathfrak{g} \longrightarrow \operatorname{End}(\mathfrak{g})
$$

defined by

$$
f(X)(Y)=\nabla(X, Y)
$$

is a linear representation (non faithful) of $\mathfrak{g}$ satisfying

$$
f(X)(Y)-f(Y)(X)=[X, Y] .
$$


REMARK 1.2. The adjoint representation $\tilde{f}$ of $\mathfrak{g}$ satisfies

$$
\tilde{f}(X)(Y)-\tilde{f}(Y)(X)=2[X, Y]
$$

and cannot correspond to an affine structure.

1.2. Classical examples of affine structures. (i) Let $\mathfrak{g}$ be the $n$-dimensional abelian Lie algebra. Then the representation

$$
f: \mathfrak{g} \longrightarrow \operatorname{End}(\mathfrak{g}), \quad X \longmapsto f(X)=0
$$

defines an affine structure.

(ii) Let $\mathfrak{g}$ be a $2 p$-dimensional Lie algebra endowed with a symplectic form

$$
\theta \in \Lambda^{2} \mathfrak{g}^{*} \text { such that } d \theta=0
$$

with

$$
d \theta(X, Y, Z)=\theta(X,[Y, Z])+\theta(Y,[Z, X])+\theta(Z,[X, Y])
$$

For every $X \in \mathfrak{g}$ we can define a unique endomorphism $\nabla_{X}$ by

$$
\theta(\operatorname{ad} X(Y), Z)=-\theta\left(Y, \nabla_{X}(Z)\right) .
$$

Then $\nabla(X, Y)=\nabla_{X}(Y)$ is an affine structure on $\mathfrak{g}$.

(iii) Following the work of Benoist [1] and Burde [2, 3, 4], we know that there exists a nilpotent Lie algebra without affine structures.

1.3. Faithful representations associated to an affine structure. Let $\nabla$ be an affine structure on an $n$-dimensional Lie algebra $\mathfrak{g}$. We consider the $(n+1)$-dimensional linear representation given by

$$
\rho: \mathfrak{g} \longrightarrow \operatorname{End}(\mathfrak{g} \bigoplus \mathbb{R})
$$

given by

$$
\rho(X):(Y, t) \longmapsto(\nabla(X, Y)+t X, 0) .
$$

It is easy to verify that $\rho$ is a faithful representation of dimension $n+1$.

We can note that this representation gives also an affine representation of $\mathfrak{g}$

$$
\psi: \mathfrak{g} \longrightarrow \operatorname{aff}\left(\mathbb{R}^{n}\right), \quad X \longmapsto\left(\begin{array}{cc}
A(X) & X \\
0 & 0
\end{array}\right),
$$

where $A(X)$ is the matrix of the endomorphisms $\nabla_{X}: Y \rightarrow \nabla(X, Y)$ in a given basis.

Definition 1.3. We say that the representation $\rho$ is nilpotent if the endomorphisms $\rho(X)$ are nilpotent for every $X$ in $\mathfrak{g}$.

Proposition 1.4. Suppose that $\mathfrak{g}$ is a complex non-abelian indecomposable nilpotent Lie algebra and let $\rho$ be a faithful representation of $\mathfrak{g}$. Then there exists a faithful nilpotent representation of the same dimension. 
Proof. Consider the $\mathfrak{g}$-module $M$ associated to $\rho$. Then, as $\mathfrak{g}$ is nilpotent, $M$ can be decomposed as

$$
M=\bigoplus_{i=1}^{k} M_{\lambda_{i}},
$$

where $M_{\lambda_{i}}$ is a $\mathfrak{g}$-submodule, and the $\lambda_{i}$ are linear forms on $\mathfrak{g}$. For all $X \in \mathfrak{g}$, the restriction of $\rho(X)$ to $M_{i}$ is in the following form:

$$
\left(\begin{array}{cccc}
\lambda_{i}(X) & * & \cdots & * \\
0 & \ddots & \ddots & \vdots \\
\vdots & \ddots & \ddots & * \\
0 & \cdots & 0 & \lambda_{i}(X)
\end{array}\right) .
$$

Let $\mathbb{K}_{\lambda_{i}}$ be the one-dimensional $\mathfrak{g}$-module defined by

$$
\mu: X \in \mathfrak{g} \longrightarrow \mu(X) \in \text { End } \mathbb{K}
$$

with

$$
\mu(X)(a)=\rho(X)(a)=\lambda_{i}(X) a .
$$

The tensor product $M_{\lambda_{i}} \otimes \mathbb{K}_{-\lambda_{i}}$ is the $\mathfrak{g}$-module associated to

$$
X \cdot(Y \otimes a)=\rho(X)(Y) \otimes a-Y \otimes \lambda_{i}(X) a .
$$

Then $\widetilde{M}=\bigoplus\left(M_{\lambda_{i}} \otimes K_{-\lambda_{i}}\right)$ is a nilpotent $\mathfrak{g}$-module. We prove that $\widetilde{M}$ is faithful. Recall that a representation $\rho$ of $\mathfrak{g}$ is faithful if and only if $\rho(Z) \neq 0$ for every $Z \neq 0 \in Z(\mathfrak{g})$. Consider $X \neq 0 \in Z(\mathfrak{g})$. If $\tilde{\rho}(X)=0$, then $\rho(X)$ is a diagonal endomorphism. By hypothesis $\mathfrak{g} \neq Z(\mathfrak{g})$ and there is $i \geq 1$ such that $X \in \mathfrak{b}^{i}(\mathfrak{g})$, we have

$$
X=\sum_{j} a_{j}\left[Y_{j}, Z_{j}\right]
$$

with $Y_{j} \in \mathscr{C}^{i-1}(\mathfrak{g})$ and $Z_{j} \in \mathfrak{g}$. The endomorphisms $\rho\left(Y_{j}\right) \rho\left(Z_{j}\right)-\rho\left(Z_{j}\right) \rho\left(Y_{j}\right)$ are nilpotent and the eigenvalues of $\rho(X)$ are 0 . Thus $\rho(X)=0$ and $\rho$ is not faithful. Then $\tilde{\rho}(X) \neq 0$ and $\tilde{\rho}$ is a faithful representation.

\section{Affine structures on Lie algebra of maximal class}

\subsection{Definition}

Definition 2.1. An $n$-dimensional nilpotent Lie algebra $\mathfrak{g}$ is called of maximal class if the smallest $k$ such that $\mathscr{C}^{k} \mathfrak{g}=\{0\}$ is equal to $n-1$.

In this case the descending sequence is

$$
\mathfrak{g} \supset \mathscr{b}^{1} \mathfrak{g} \supset \cdots \supset \mathscr{C}^{n-2} \mathfrak{g} \supset\{0\}=\mathscr{C}^{n-1} \mathfrak{g}
$$

and we have

$$
\begin{gathered}
\operatorname{dim} \mathfrak{G}^{1} \mathfrak{g}=n-2, \\
\operatorname{dim} \mathfrak{C}^{i} \mathfrak{g}=n-i-1, \quad \text { for } i=1, \ldots, n-1 .
\end{gathered}
$$


EXAMPLE 2.2. The $n$-dimensional nilpotent Lie algebra $L_{n}$ defined by

$$
\left[X_{1}, X_{i}\right]=X_{i+1} \quad \text { for } i \in\{2, \ldots, n-1\}
$$

is of maximal class.

We can note that any Lie algebra of maximal class is a linear deformation of $L_{n}$ [5].

2.2. On non-nilpotent affine structure. Let $\mathfrak{g}$ be an $n$-dimensional Lie algebra of maximal class provided with an affine structure $\nabla$. Let $\rho$ be the $(n+1)$-dimensional faithful representation associated to $\nabla$ and we note that $M=\mathfrak{g} \oplus \mathbb{C}$ is the corresponding complex $\mathfrak{g}$-module. As $\mathfrak{g}$ is of maximal class, its decomposition has one of the following forms

$$
M=M_{0}, \quad M \text { is irreducible, }
$$

or

$$
M=M_{0} \bigoplus M_{\lambda}, \quad \lambda \neq 0
$$

For a general faithful representation, we call characteristic the ordered sequence of the dimensions of the irreducible submodules. In the case of maximal class we have $c(\rho)=(n+1)$ or $(n, 1)$ or $(n-1,1,1)$ or $(n-1,2)$. In fact, the maximal class of $\mathfrak{g}$ implies that there exists an irreducible submodule of dimension greater than or equal to $n-1$. More generally, if the characteristic sequence of a nilpotent Lie algebra is equal to $\left(c_{1}, \ldots, c_{p}, 1\right)$ (see [5]) then for every faithful representation $\rho$ we have $c(\rho)=\left(d_{1}, \ldots, d_{q}\right)$ with $d_{1} \geq c_{1}$.

THEOREM 2.3. Let $\mathfrak{g}$ be the Lie algebra of the maximal class $L_{n}$. Then there are faithful g-modules which are not nilpotent.

Proof. Consider the following representation given by the matrices $\rho\left(X_{i}\right)$ where $\left\{X_{1}, \ldots, X_{n}\right\}$ is a basis of $\mathfrak{g}$

$$
\rho\left(X_{1}\right)=\left(\begin{array}{ccccccccc}
a & a & 0 & \cdots & \cdots & & & 0 & 1 \\
a & a & 0 & & & & & \vdots & 0 \\
0 & 0 & 0 & & & & & 0 & 0 \\
\vdots & \ddots & \frac{1}{2} & \ddots & & & & \vdots & 0 \\
\vdots & & \ddots & \ddots & \ddots & & & \vdots & 0 \\
\vdots & & & \ddots & \frac{i-3}{i-2} & \ddots & & \vdots & 0 \\
0 & 0 & & & \ddots & \ddots & \ddots & \vdots & 0 \\
\alpha & \beta & 0 & \cdots & \cdots & 0 & \frac{n-3}{n-2} & 0 & 0 \\
0 & 0 & 0 & 0 & 0 & 0 & 0 & 0 & 0
\end{array}\right),
$$




$$
\rho\left(X_{2}\right)=\left(\begin{array}{ccccccccc}
a & a & 0 & \cdots & \cdots & & \cdots & 0 & 0 \\
a & a & 0 & & & & & \vdots & 1 \\
-1 & 1 & 0 & & & & & 0 & 0 \\
0 & 0 & \frac{1}{2} & \ddots & & & & \vdots & 0 \\
\vdots & & \ddots & \ddots & \ddots & & & \vdots & 0 \\
\vdots & & & \ddots & \frac{1}{i-2} & \ddots & & \vdots & 0 \\
0 & 0 & & & \ddots & \ddots & \ddots & \vdots & 0 \\
\beta & \alpha & 0 & \cdots & \cdots & \cdots & \frac{1}{n-2} & 0 & 0 \\
0 & 0 & 0 & 0 & 0 & 0 & 0 & 0 & 0
\end{array}\right),
$$

and for $j \geq 3$ the endomorphisms $\rho\left(X_{j}\right)$ satisfy

$$
\begin{gathered}
\rho\left(X_{j}\right)\left(e_{1}\right)=-\frac{1}{j-1} e_{j+1}, \\
\rho\left(X_{j}\right)\left(e_{2}\right)=\frac{1}{j-1} e_{j+1}, \\
\rho\left(X_{j}\right)\left(e_{3}\right)=\frac{1}{j(j-1)} e_{j+2}, \\
\vdots \\
\rho\left(X_{j}\right)\left(e_{i-j+1}\right)=\frac{(j-2) !(i-j-1) !}{(i-2) !} e_{i}, \quad i=j-2, \ldots, n, \\
\rho\left(X_{j}\right)\left(e_{i-j+1}\right)=0, \quad i=n+1, \ldots, n+j-1, \\
\rho\left(X_{j}\right)\left(e_{n+1}\right)=e_{j},
\end{gathered}
$$

where $\left\{e_{1}, \ldots, e_{n}, e_{n+1}\right\}$ is the basis given by $e_{i}=\left(X_{i}, 0\right)$ and $e_{n+1}=(0,1)$. We easily verify that these matrices describe a nonnilpotent faithful representation.

2.3. Noncomplete affine structure on $L_{n}$. The previous representation is associated to an affine structure on the Lie algebra $L_{n}$ given by

$$
\nabla\left(X_{i}, Y\right)=\rho\left(X_{i}\right)(Y, 0)
$$

where $L_{n}$ is identified to the $n$-dimensional first factor of the $(n+1)$-dimensional faithful module. This affine structure is complete if and only if the endomorphisms $R_{X} \in \operatorname{End}(\mathfrak{g})$ defined by

$$
R_{X}(Y)=\nabla(Y, X)
$$


are nilpotent for all $X \in \mathfrak{g}$ (see [6]). But the matrix of $R_{X_{1}}$ has the form

$$
\left(\begin{array}{cccccccc}
a & a & 0 & \cdots & 0 & \cdots & 0 & 0 \\
a & a & & & \vdots & & \vdots & 0 \\
0 & -1 & & & \vdots & & \vdots & 0 \\
0 & 0 & -\frac{1}{2} & \cdots & 0 & \cdots & 0 & 1 \\
\vdots & \vdots & 0 & \ddots & & \cdots & \vdots & 0 \\
0 & 0 & \vdots & \ddots & -\frac{1}{j-1} & & \vdots & 0 \\
\alpha & \beta & \vdots & \cdots & \ddots & \ddots & 0 & 0 \\
0 & 0 & 0 & 0 & 0 & 0 & -\frac{1}{n-2} & 0
\end{array}\right) .
$$

Its trace is $2 a$ and for $a \neq 0$ it is not nilpotent. We have proved the following proposition.

Proposition 2.4. There exist affine structures on the Lie algebra of maximal class $L_{n}$ which are noncomplete.

REMARK 2.5. The most simple example is on $\operatorname{dim} 3$ and concerns the Heisenberg algebra. We find a nonnilpotent faithful representation associated to the noncomplete affine structure given by

$$
\nabla_{X_{1}}=\left(\begin{array}{ccc}
a & a & 0 \\
a & a & 0 \\
\alpha & \beta & 0
\end{array}\right), \quad \nabla_{X_{2}}=\left(\begin{array}{ccc}
a & a & 0 \\
a & a & 0 \\
\beta-1 & \alpha+1 & 0
\end{array}\right), \quad \nabla_{X_{3}}=\left(\begin{array}{ccc}
0 & 0 & 0 \\
0 & 0 & 0 \\
0 & 0 & 0
\end{array}\right),
$$

where $X_{1}, X_{2}$, and $X_{3}$ are a basis of $H_{3}$ satisfying $\left[X_{1}, X_{2}\right]=X_{3}$ and $\nabla_{X_{i}}$ the endomorphisms of $\mathfrak{g}$ given by

$$
\nabla_{X_{i}}\left(X_{j}\right)=\nabla\left(X_{i}, X_{j}\right)
$$

The affine representation is written as

$$
\left(\begin{array}{cccc}
a\left(x_{1}+x_{2}\right) & a\left(x_{1}+x_{2}\right) & 0 & x_{1} \\
a\left(x_{1}+x_{2}\right) & a\left(x_{1}+x_{2}\right) & 0 & x_{2} \\
\alpha x_{1}+(\beta-1) x_{2} & \beta x_{1}+(\alpha+1) x_{2} & 0 & x_{3} \\
0 & 0 & 0 & 0
\end{array}\right) .
$$

\section{REFERENCES}

[1] Y. Benoist, Une nilvariété non affine [A non-affine nilvariety], J. Differential Geom. 41 (1995), no. 1, 21-52 (French).

[2] D. Burde, Affine structures on nilmanifolds, Internat. J. Math. 7 (1996), no. 5, 599-616.

[3] _ Left-invariant affine structures on reductive Lie groups, J. Algebra 181 (1996), no. 3, 884-902.

[4] _ _ Simple left-symmetric algebras with solvable Lie algebra, Manuscripta Math. 95 (1998), no. 3, 397-411. 
[5] M. Goze and Y. Khakimdjanov, Nilpotent Lie Algebras, Mathematics and Its Applications, vol. 361, Kluwer Academic Publishers Group, Dordrecht, 1996.

[6] J. Helmstetter, Radical d'une algèbre symétrique à gauche, Ann. Inst. Fourier (Grenoble) 29 (1979), no. 4, 17-35 (French).

E. Remm: Faculté des Sciences et Techniques, 4, Rue des Frères Lumière, F. 68093 Mulhouse Cedex, France

E-mail address: e. remm@univ-mu 7 house. fr

Michel Goze: Faculté des Sciences et Techniques, 4, Rue des Frères Lumière, F. 68093 Mulhouse CEDEX, FrANCE

E-mail address: m. goze@univ-muThouse.fr 


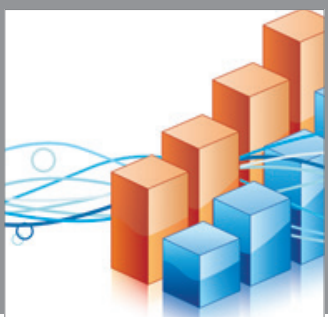

Advances in

Operations Research

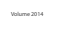

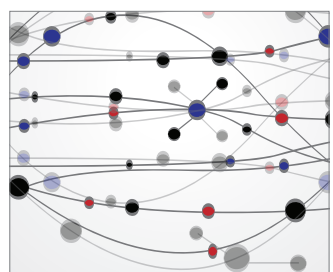

\section{The Scientific} World Journal
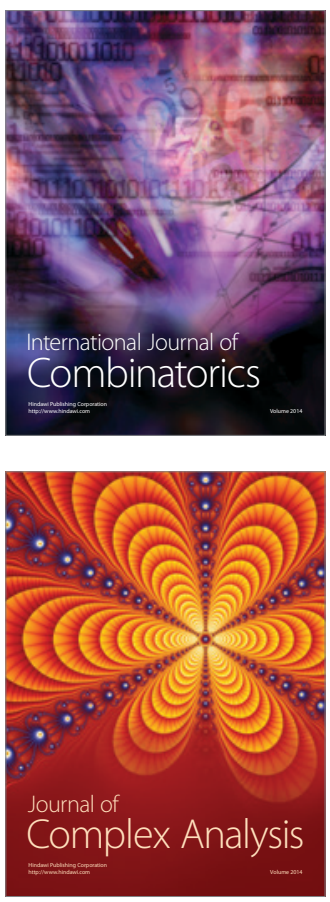

International Journal of

Mathematics and

Mathematical

Sciences
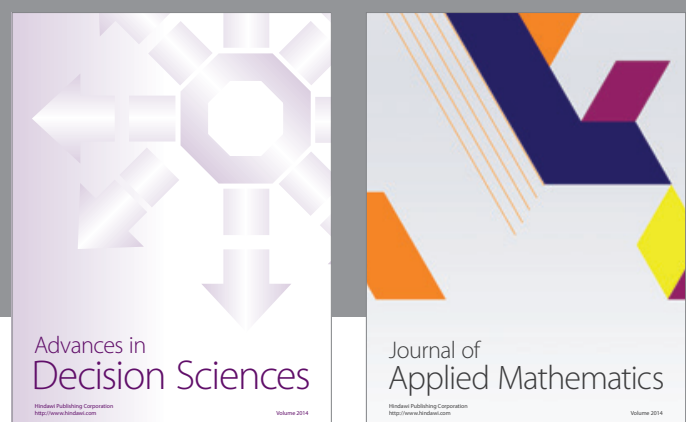

Journal of

Applied Mathematics
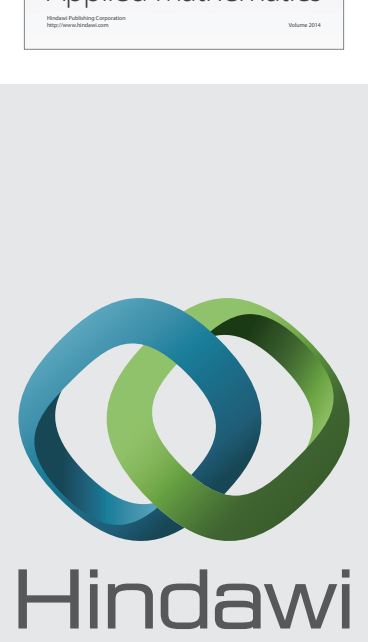

Submit your manuscripts at http://www.hindawi.com
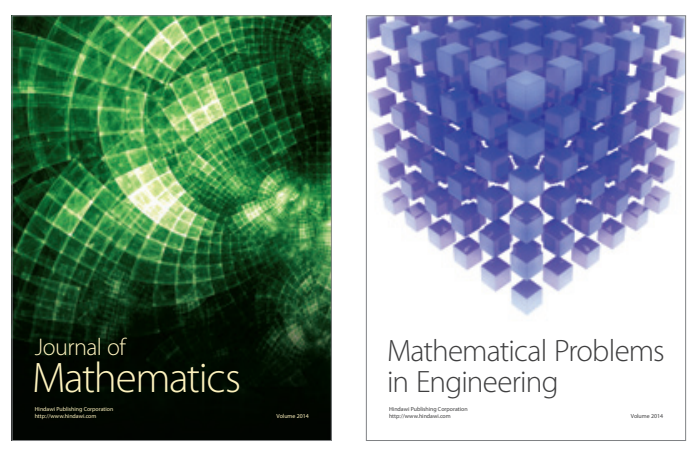

Mathematical Problems in Engineering
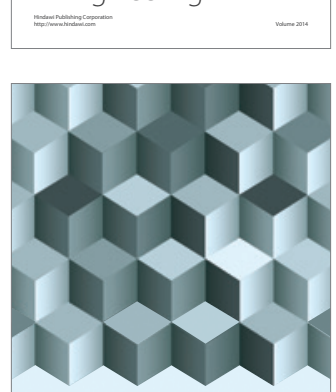

Journal of

Function Spaces
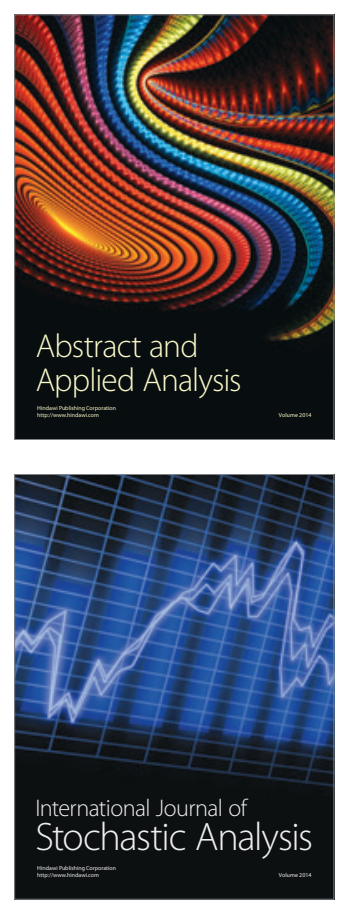

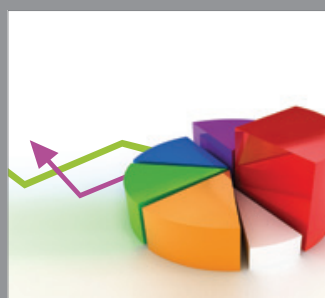

ournal of

Probability and Statistics

Promensencen
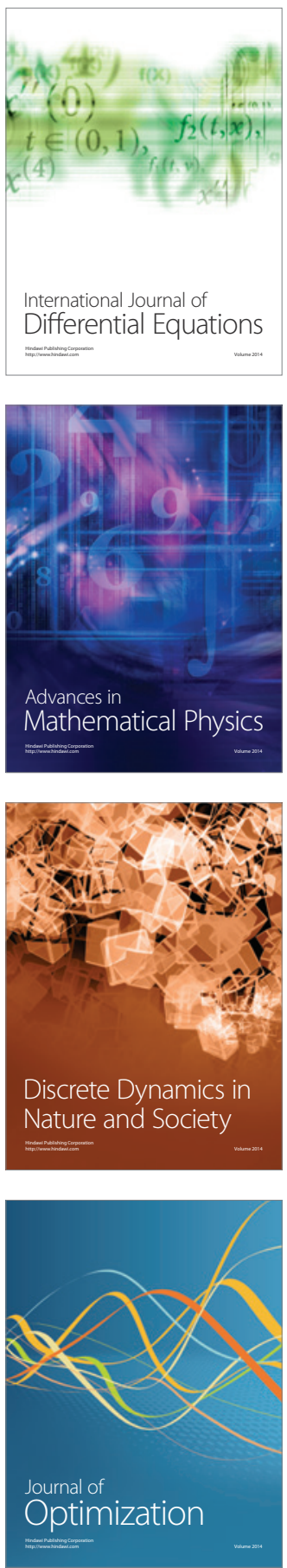